\title{
« Ni putes ni soumises »
}

Émergence et politisation d'un mouvement de femmes dans l'espace public

Emergence and Politicisation of a Women's Movement within the Public Space:

«Ni putes ni soumises»

\section{Sylvie Thiéblemont-Dollet}

\section{(2) OpenEdition}

\section{Journals}

Édition électronique

URL : http://journals.openedition.org/questionsdecommunication/4060

DOI : 10.4000/questionsdecommunication.4060

ISSN : 2259-8901

\section{Éditeur}

Presses universitaires de Lorraine

\section{Édition imprimée}

Date de publication : 30 juin 2005

Pagination : 105-120

ISBN : 978-2-86480-859-6

ISSN : 1633-5961

\section{Référence électronique}

Sylvie Thiéblemont-Dollet, « « Ni putes ni soumises » », Questions de communication [En ligne], 7| 2005, mis en ligne le 11 mai 2012, consulté le 30 avril 2019. URL : http://journals.openedition.org/ questionsdecommunication/4060 ; DOI : 10.4000/questionsdecommunication.4060 


\title{
$>$ DO SSIER
}

SYIVIE THIÉBLEMO NT-DO ШET

Université Nancy 2

Centre de recherche sur les médiations

Université Paul Verlaine-Metz

Sylvie.Thieblemont@univ-nancy2.fr

\begin{abstract}
" NI PUTES NI SO UMISES " . ÉMERGENCE ET POUTISATION D'UN MOUVEMENT

DE FEMMES DANS L'ESPACE PUBUC
\end{abstract}

Résumé. - Parce qu'elle a été élue présidente de la fédération nationale des "Maisons des Potes" (décembre 2000), qu'elle est un des membres fondateurs de l'association " $\mathrm{Ni}$ putes $\mathrm{ni}$ soumises ", Fadela Amara tend à participer à la construction de la représentation d'un mouvement de femmes dans l'espace public. De même, la façon dont certains hommes politiques et journalistes contribuent, à leur tour, à cette construction, montre que « $\mathrm{N}$ i putes ni soumises " vise un autre espace que celui qui serait purement associatif et se comporte, d'ores et déjà, comme un mouvement politique.

Mots clés. - Mouvement de femmes, immigrée, espace public, politisation, journalistes, hommes politiques. 
e travail est la prolongation d'une recherche portant sur le collectif « Ni putes ni soumises " (émanation de la Fédération nationale des « Maison des Potes ", créée en 2001), puis sur l'association éponyme constituée en avril 2003, et leur présidente Fadela Amara, sans oublier les femmes - pour la plupart anonymes - qui les ont rejoints à un moment ou à un autre. Plus particulièrement, ce qui nous intéresse est la manière dont Fadela Amara, fille d'immigrés algériens, née en France, a peu à peu construit, sur dix-huit mois environ ${ }^{1}$, ses énonciations dans la sphère publique (politique et médiatique) et comment, à l'inverse, la sphère politique (élus, représentants de partis, etc.) s'est appropriée une partie des contenus de ses discours (projet de société, propositions concrètes, etc.), parce qu'ils sont l'émanation et la synthèse de propos de femmes représentantes de l'immigration. De même, comme ils ont relié ces deux pôles, instauré une relation triangulaire et participé de la construction de l'autoreprésentation et de l'hétéro-représentation de Fadela Amara, les médias ont été intégrés à la réflexion. Cette étude résulte de l'observation et de l'analyse des différents glissements ou passages des réseaux informels aux réseaux associatifs, puis politico-médiatiques et inversement, opérés par Fadela Amara par les militants des mouvements suscités ou proches, les journalistes et les acteurs politiques. Car, volontairement ou non, elle a suscité les convoitises d'un certain nombre de journalistes et d'hommes politiques, sans doute pour des logiques de pouvoir dont elle n'a pas toujours mesuré l'ampleur, logiques reliées à des enjeux politiques nationaux importants (e.g. discussion sur le voile, rôle de Tariq Ramadan dans l'échange sur la laïcité à l'occasion du débat télévisé sur France 2 avec $\mathrm{N}$ icolas Sarkozy, le 20 novembre 2003, etc.) : on peut réellement parler ici de va-et-vient entre les courants associatif et médiaticopolitique. Dès lors, on comprendra l'utilité de se demander si Fadela Amara n'a pas été l'objet de cette instrumentalisation que de nombreuses femmes dénoncent (Pionchon, Derville, 2004), qu'elles soient acteurs politiques (élues) ou tout simplement acteurs du débat public (militantes, membres d'une association).

Par ailleurs, comme le suggère le texte de présentation de la journée d'étude du 8 mars 2004, organisée par les revues Communications, M ots et Questions de communication, l'exploration de tels phénomènes permet de comprendre et d'évaluer l'idée selon laquelle on assiste à une féminisation de la politique. C'est donc à partir des propositions de

\footnotetext{
${ }^{1}$ Cette période est constituée d'au moins trois moments clés par rapport à l'évolution du collectif : les journées de la femme des 8 mars 2003 et 2004, qui correspondent également à des manifestations importantes du collectif lui-même telles les marches des “ Ni putes ni soumises ", et leur université annuelle des 8,9 et 10 octobre 2004, dédiée à S. Bellil, auteur de Dans l'enfer des tournantes (2002), et décédée le 7 septembre. 2004.
} 
recherche, proches de celles de Simone Bonnafous (1999:59-72), que nous avons étayé notre raisonnement pour éclairer la médiatisation politique de Fadela Amara. Face au champ de l'immigration, cette même chercheuse (ibid. : 66) constate que "si le discours des acteurs politiques légitimes et institués a été fréquemment étudié, bien que de façon inégale, l'absence d'étude méthodique des discours et des pratiques médiatiques des autres acteurs de cette co-production est frappante ".Ainsi suggère-t-elle un certain nombre de questionnements (1999 :66-67) que nous nous sommes appropriés : Fadela Amara étant d'origine immigrée, comment et où s'exprime-t-elle ? À quel(s) réseau(x) participe-t-elle ?De quelles symbolisations est-elle porteuse ? Quel(s) expert(s) réquisitionne-t-elle pour tel ou tel média et telle ou telle mobilisation ?A-t-elle des liens avec des chercheurs en sciences sociales ? Et de là, enfin, comment sinscrit-elle - volontairement ou non - dans le champ de l'action médiatique et politique ?

\section{Fadela Amara et « Ni putes ni soumises » dans l'espace public masculin}

\section{Glissement $d u$ « collectif » féminin \\ au mouvement associatif}

Cette série non exhaustive de questions a permis de mettre en perspective la médiatisation effective de Fadela Amara, figure emblématique de la défense des droits les plus élémentaires des femmes des quartiers - dont elle a une certaine expérience pour avoir vécu dans la cité d'Herbet, à Clermont-Ferrand - et, depuis la sortie de son livre, Ni putes ni soumises (2003), de tout autre citoyen vivant sur le sol français. Ainsi avons-nous pu constater la construction de la mise en scène de son discours, dans un espace public, au départ " sectoriel [puisque] restreint aux citoyens mobilisés et à leurs proches [femmes des quartiers, militantes], aux autorités mises en cause [élus locaux représentants de la République], aux spectateurs de hasard ou captifs [travailleurs sociaux, formateurs, policiers, religieux, chercheurs, etc.] et $\mathrm{au}(\mathrm{x})$ journaliste(s) localier(s) 》 (Favre, $1999: 148)$, mais ouvrant, peu à peu, à un « espace public de discussion " plus large (ibid. :150). D'une part, par de nombreuses actions militantes servant à faire connaître le mouvement et/ou destinées au bien-être et à l'amélioration des conditions de vie des femmes, et d'autre part, par les déplacements dans toutes les villes de France lors des "Marches des femmes contre les ghettos et pour l'égalité " (01/02/03-08/03/03, 31/01/04-06/03/04), incluant systématiquement des réunions publiques relayées par les quotidiens et les télévisions généralistes de la presse régionale et 
nationale (Thiéblemont-Dollet, $2003: 120-124$ ), Fadela Amara a contribué à la construction d'un " tissu social serré et continu " (Favre, 1999 : 148). Autrement dit, dès lors qu'elle a été élue en 2000 présidente de la Fédération nationale des «Maisons des Potes » (qui comprend près de 300 associations de quartier), elle a donné un nouveau souffle à ce réseau formel, en raison des assises juridiques et administratives de ce dernier (association loi 1901) et en sollicitant les réseaux informels qui, pour certains, avaient été à l'origine de la fédération susdite ou d'autres associations inscrites dans la même mouvance. Dans le dossier "Réseaux sociaux en migrations " de la revue Hommes et migrations, Marie-Antoinette $\mathrm{Hily}$ et $\mathrm{W}$ illiam Berthomière (2004:9) parlent effectivement, pour le cas des courants migratoires, de " double démarche [consistant] à utiliser, une législation existante (loi de 1901), pour organiser les réseaux informels ". Ce qui correspond à la manière dont s'est constituée et a agi la Fédération nationale des Maisons des Potes, lorsqu'elle a pris corps officiellement, en 1988, à la suite des luttes et des marches (La Marche des Beurs de 1983 organisée par l'association SO S Avenir-Minguettes ;Convergence 1984 ; Divergence 1985) nées de différents collectifs et associations issus de l'immigration, plus ou moins organisés et concurrents (SOS Racisme, novembre 1984 ; France-Plus, printemps 1985)².

La stratégie a été la même concernant le collectif « $\mathrm{Ni}$ putes ni soumises " qui, à partir de la démultiplication de la parole de femmes réunies pour la même cause, a glissé en avril 2003 vers une forme structurelle reconnue par les institutions, celle de l'association loi 1901. De fait, après avoir été chargé de fournir - provisoirement et un tant soit peu - des réponses au problème posé, et après avoir tenté de démontrer que les " profanes " pouvaient intéresser les décideurs et les experts (concept de démocratie technique défendu par Barthes, Callon, Lascoumes, $2001: 16$ ), le collectif transformé en association s'est donné davantage de légitimité. Dès lors, cette mutation lui a permis d'associer plus souvent aux paroles de profanes (témoignages, confidences, etc.) celles d'experts (colloques, universités, etc.), d'assumer une vraie fonction de relais, de médiation et de négociation (entre les citoyen(ne)s qui ont parlé et les décideurs) et lui a donné, par conséquent, une autre visibilité. De la sorte, les espaces publics locaux ou " mosaiqques " (François, Neveu, 1999 : 48-52), greffés autour du collectif « $\mathrm{Ni}$ putes ni soumises ", puis de l'association du même nom, ont produit des débats argumentés qui se sont intégrés dans le champ politique, lui-même « transmutateur des problèmes " (Favre, $1999: 149$ ).

\footnotetext{
2 À ce sujet, les contributions d'acteurs (D elorme, 1985 ; Malik, 1990 ;Amara, 2003) ou d'auteurs (Bouamama, 1994 ; Gastaut, 2000) sont suffisamment fournies en explications et détails pour ne pas y revenir.
} 


\section{Lémergence d'un projet politique asexué}

Comme le souligne Pierre Favre (1999:149), de par sa position, l'homme politique redéfinit et déplace les problèmes $0 \mathrm{r}$, en glissant de la création de l'association « Ni putes ni soumises 》 - invention auparavant « inimaginable ou inexprimable dans l'espace public " (François, Neveu, $1999: 47$ ) parce que relevant du genre féminin - à l'écriture d'un ouvrage du même nom, avec la collaboration de Sylvia Zappi, journaliste au quotidien Le Monde, Fadela Amara a élargi son combat, initialement centré sur les femmes de quartier, à un manifeste de type politique. En ce sens, elle a procédé à une forme de mutation de l'idéologie de départ (reconnaissance à part entière de la femme dans la société française quels que soient ses origines, son travail, son lieu de vie, etc.) qu'elle a construite à partir de constats émanant de témoignages (Le Livre Blanc des Femmes de Quartier, mars 2002 ; les paroles ou confidences recueillies à l'occasion des marches et des différentes réunions locales ou nationales) et d'expertises (e.g. implication de chercheurs tels que Michel Fize, Hélène O rain, Daniel Welzer-Lang, sociologues ; Françoise Picq, chargée de mission à l'Égalité des chances) sur le quotidien de ceux et celles qui vivent au cœur des cités et des banlieues

Ainsi son livre est-il le point d'ancrage de l'évolution d'un « problème public " $^{3}$,tel qu'Érik N eveu le définit (1999:41-42), dans lequel figure une étape intermédiaire qu'est l'ouverture du mouvement à toutes les femmes de la société française. À l'issue de la première "Marche des femmes contre les ghettos et pour l'égalité ", de nombreuses femmes, ne vivant ni dans les cités, ni dans la précarité, sont venues à la rencontre des membres du collectif et ont fait part de leur sort, aussi peu enviable que celui des cités. C'est à ce moment que « Ni putes ni soumises "sinscrit fortement dans ce que François de Singly (2003:176-77) qualifie de « démocratie participative, [à savoir] une régulation qui parte plus du bas (bottom up) que du haut (top down) ", et qui réhabilite la fonction de la société civile. Autrement dit, chaque individu devient partie prenante de son destin individuel et du destin de la communauté dans la ville, dès lors qu'il est militant ou sympathisant d'un mouvement, voire tout simplement conscient de son devoir d'agir pour lui-même (sil est victime) ou pour l'autre (par devoir envers les victimes) ${ }^{4}$. C'est de cette façon que, au cours des mois,

\footnotetext{
${ }^{3}$ É. Neveu définit la notion de " problème public " (social problem) comme "la transformation d'un fait social quelconque en enjeu de débat public et/ou d'intervention étatique [...] sill est constitué par l'action volontariste de divers opérateurs ".

${ }^{4}$ Cette manière d'être ou de penser l'individu correspond à la définition de la notion d'empowerment, proposée par J Donzelot et C. Mével (2002:86), qui ne peut avoir de " sens que si et seulement si, les autorités politiques et les experts considèrent les individus ordinaires comme doués d'une capacité réflexive " (de Singly, 2003 :177). Et même si, en France, ce modèle ne fait pas partie des nouveaux processus de participation, il est à l'état embryonnaire et peut contribuer aux futurs projets en terme de politiques de la ville.
} 
le projet de société porté par Fadela Amara et ses militant(e)s se métamorphose en un projet plus large et "constructif " (Amara, 2003 :130), chacun devant pouvoir y puiser des références universelles (droits de l'homme, valeurs de la République) ${ }^{5}$. De la même manière, il n'implique plus directement les hommes dans les difficultés que les femmes avaient dénoncées dans un premier temps, mais au contraire, il les transforme, à leur tour, en victimes d'un système discriminatoire, en partie lié au désengagement des représentants (élus) de la République : inégalités des salaires, emplois non attribués en raison de l'appar tenance identitaire d'origine, politiques d'exclusion engendrant des attitudes délinquantes, etc. Pour exemple marquant de cette transformation, on peut citer la manifestation nationale du 6 mars 2004, clôturant la deuxième marche, qui précise, en guise d'intitulé, qu'en plus de la défense des droits des femmes, elle appelle à la laïcité, la mixité et l'égalité pour tous ${ }^{6}$.

De plus en plus, il apparaît que les militants de " Ni putes ni soumises" cherchent à gommer toute éventuelle " appartenance [ou] rattachement à une communauté englobante " (de Singly, 2003 : 89), féministe ou autre :par exemple, maghrébine, femme de cité, etc. Cette position n'est pas hasardeuse et elle semble reliée aux critiques des autres mouvements féministes qui n'entendent pas de la sorte le combat des femmes. Du coup, Fadela Amara et l'une de ses porteparole les plus médiatisées, Samira Bellil - parce qu'elle est auteure du livre Dans l'enfer des tournantes (2002) qui a fait scandale - occupent, dès que faire se peut, la scène médiatique pour dire que leurs revendications et propositions n'ont rien à voir avec le féminisme en place ou reconnu. À l'inverse, au vu de la montée en puissance de l'association " $\mathrm{Ni}$ putes ni soumises ", certaines féministes (e.g. " Les Chiennes de garde ", le MLF) et intellectuelles (Élisabeth Badinter, Marcela lacub) tentent de réduire, également par médias interposés (interviews dans la presse, droits de réponse, etc.), les revendications de leurs consœurs à un simple réquisitoire des délits et des crimes de droit commun (e.g. meurtre de Sohane ${ }^{7}$ ), précisant ainsi que ces requêtes

\footnotetext{
${ }^{5}$ Ce projet est détaillé dans le livre de F. Amara (2003:124-138) ; mais, il est régulièrement mis à jour sur les sites http:/www.macite.net et http $: /$ www.niputesnisoumisescom. Les trois grands aspects en sont : droit à l'éducation sexuelle pour tous ; droit à l'éducation civique ; droit à la sécurité pour tous.

${ }^{6}$ Appel à la manifestation du mouvement “ $\mathrm{N}$ i putes ni soumises ", 6 mars 2004, place de la République, Paris, à l'issue de la seconde marche des femmes (Pour la défense des droits des femmes. Pour la laïcité, la mixité, l'égalité) mis en ligne sur les sites : http:/www.macite.net/home/ et http:/www.niputesnisoumises.com.

${ }^{7}$ Sohane est morte le 4 octobre 2002, brûlée vive dans un local à poubelles d'une cave de la cité Balzac, à Vitry-sur-Seine, par un garçon de 19 ans. Depuis, sa sœur, Kahina, dénonce la barbarie de cet acte et refuse de senfermer dans le silence.
} 
relèvent uniquement de la police et non pas d'un mouvement d'émancipation des femmes, et dénoncent, par la même occasion l'usage de la " rhétorique de la victimisation " de ces mouvements. Ce qui, à lire Fausse Route d'Élisabeth Badinter (2003), revient à dire que de tels mouvements tendent à la simplification (victimisme ou différentialisme) et nuisent à leur propre cause. Sauf que, même si elle en a fait usage puisque « choisir de sériger en victime pour faire parler de soi est [...] un choix particulièrement en phase avec une société où la victimité est devenue une forme de légitimation de soi la plus aboutie " (Abel, 2004), l'association “ N i putes ni soumises » ne se cantonne pas à cette seule rhétorique : elle s'inscrit dans d'autres stratégies rhétoriques (Thiéblemont-D ollet, 2003) et politiques. En outre, si l'on reprend $X Y$. De l'identité masculine de la même philosophe $(1992,2004)$, le combat de départ des « $\mathrm{Ni}$ putes ni soumises " rappelle pourtant bien celui des Précieuses françaises des années 1650, qu'elle cite et défend pour avoir été la première expression d'une " remise en question du rôle des hommes et de l'identité masculine, [...] née en réaction à la grossièreté des hommes de la cour d'Henri IV et de ceux de la Fronde " (1986 : 25-26). En effet, le but de ce mouvement est de remettre en cause l'autoritarisme du père et du mari, de s'opposer au mariage arrangé et de revendiquer le droit à la dignité et au savoir. Certes, la « Précieuse » qui n'a pas bonne presse dans la mémoire collective suite à la levée de boucliers qu'elle a suscitée, notamment dans la littérature, avec entre autres œuvres la caricaturant, Les Précieuses ridicules de Molière (1659) est généralement issue de la classe dominante, alors que la militante de " $\mathrm{N}$ i putes ni soumises " l'est rarement. Mais peu importe. Dans un cas comme dans l'autre, ces figures se situent dans des démarches similaires, malgré les siècles qui les séparent. Et ce n'est pas par l'opposition aux hommes (soit le différentialisme) qu'elles ont pris corps dans l'espace public, mais par des propositions de savoir-faire et savoir-être adaptables aux deux sexes (les effets suite à leurs actions). Enfin, si le "Précieux 》 a existé et adhéré à la cause défendue, des sympathisants et militants ont adhéré à « $\mathrm{Ni}$ putes ni soumises" et participent à son fonctionnement ${ }^{9}$. Ce qui explique, dès lors, la posture que Fadela Amara revendique, celle de "l'identité fluide [...] multidimensionnelle » vs unidimensionnelle (de Singly, $2003: 84,94$ ) qui, outre son titre de représentante du mouvement, lui offre la possibilité de marquer son propre espace dans l'espace politique, d'afficher une identité ouverte à géométrie variable et de combiner, à son tour, différentes fonctions, savoir-faire et savoir-être. Ainsi a-t-elle établi des partenariats dialogiques

\footnotetext{
¿ J Savigneau, "Élisabeth Badinter dénonce la «rhétorique de victimisation» de certains mouvements ", Le Monde (27-28/04/03).

${ }^{9}$ Certains adhérents sont à des postes clé : par exemple, M. Abdi est le secrétaire général de l'association.
} 
(relations régulières entre les témoins, militants, élus associatifs, experts et décideurs), le but étant de construire un mouvement citoyen devant " rester en dehors de toute forme d'instrumentalisation " (Amara, $2003: 130$ ), au sein duquel les revendications transcendent les classes sociales et dépassent les clivages politiques.

\section{Lappropriation du mouvement par l'espace médiatico-politique}

\section{Fadela Amara dans l'arène politico-médiatique : pourquoi et comment?}

En occupant de plus en plus souvent la scène politico-médiatique, Fadela Amara et l'association " $\mathrm{N}$ i putes ni soumises " produisent ce que nous avons appelé des " discours sur ", c'est-à-dire des discours politiques relayés par les médias - qui prennent position, d'une manière ou d'une autre, sur le projet défendu. Malgré la présence, même très assidue, de militants masculins, ce projet est mené par de nombreuses femmes originaires des banlieues, pour la plupart issues de l'immigration, et de fait, il retient l'attention pour ce que peuvent représenter ces trois qualificatifs : femme, issue de l'immigration (ou immigrée), vivant (ou ayant vécu) dans une cité. Ces critères participent certainement de la perversité du système politique du fait que ce dernier peut cacher des manœuvres indignes : la femme en tant que "débatteuse " publique est mise sur le devant de la scène pour servir l'image progressiste d'un parti ou d'un mouvement. Pur alibi ou simple figurante, elle est le jouet d'enjeux dont elle n'a pas systématiquement connaissance (Pionchon, Derville, 2004 :73-106), même si elle s'en défend comme le fait Fadela Amara. En outre, si le constat est que, au gré des semaines et des mois, les manifestations mises au point par la Fédération nationale des Maisons des Potes, puis par " $\mathrm{Ni}$ putes ni soumises", en raison de leur importance quantitative et politique, font partie de celles dont "les médias ne peuvent pas ne pas choisir de ne pas rendre compte, [...] qu'elles sont attendues et se transforment en "événement annoncé, attendu, scruté" 》 (Favre, 1999 : 145 ; voir conférences de presse, annonces télévisées dans les journaux télévisés, mailings, lettre de macite.net, etc.), qu'en est-il du rôle de Fadela Amara ?A-t-elle œuvré pour la stratégie de " retournement de stigmate " (Pionchon, Derville, 2004 :102), c'est-à-dire a-t-elle usé de sa triple propriété - femme, issue de l'immigration, ayant vécu dans une banlieue - comme atout et ressource pour se faire entendre et entrer en politique ?Au vu de son rôle de militante de base, de présidente de la Fédération nationale des "Maisons des Potes", de fondatrice du collectif « Ni putes ni 
soumises ", de présidente de l'association qui en résulte, et enfin des propos qu'elle tient régulièrement en public, elle semble s'inscrire dans cette démarche qui n'a rien de péjoratif : sans cela, elle n'aurait jamais pu devenir un porte-parole collectif, ni prendre racine dans l'espace public politique.

En d'autres termes, elle a gagné en visibilité et n'est pas seulement la figure emblématique d'un mouvement militant et particulier, mais la représentante de courants de pensée dont les politiques, élus ou non (c'est-à-dire militants d'un parti, d'un syndicat, etc.), " ne peuvent pas ne pas choisir de ne pas rendre compte " (Favre, 1999 : 145) ou reprendre à leur compte, à l'instar des médias. Dès lors, en raison du créneau des revendications choisies, de porte-parole unique, elle devient un « moyen tactique » parmi d'autres que les politiques dans leur ensemble (sauf les représentants d'extrême droite) associent à leurs entreprises ou revendications. C'est une stratégie que l'historienne Michelle Perrot $(2001 ; 2002)$ a clairement expliquée : s'il y a crispation religieuse ou identitaire (e.g. le débat sur le port du voile en France), les femmes sont, et ont toujours été, un enjeu symbolique et de pouvoir. Cela revient à dire que les revendications des femmes, longtemps condamnées ou méprisées par les médias, sont devenues objet de succès ou de conquête, dès lors que les combats menés ont été gagnés, reconnus ou porteurs d'éventuels suffrages.

De même, au regard de la sphère politique, de telles demandes émanant de la vie privée (conjugalité, santé, famille, etc.) étalée au sein de l'arène publique, correspondent à une problématique devenue incontournable. Ce que anine Mossuz-Lavau (2004 : 24), co-auteur de Quand les femmes s'en mêlent. Genre et pouvoir, souligne en écrivant que " les Françaises aujourd'hui, du fait de leur situation historique et sociologique, ont une expertise différente, liée au fait qu'elles sont en charnière à la fois du public et du privé alors que les hommes sont nettement moins insérés dans le privé. Elles peuvent donc dispenser un point de vue qui restitue le fait qu'elles soient parfois plus en prise qu'eux sur nombre de problèmes qui se posent dans la société » actuelle et de la sorte, elle invite à repenser la fonction politique de la femme dans une perspective de genre ou de " sexe social ".

II n'est donc pas surprenant de voir des hommes politiques s'intéresser à Fadela Amara qui présente et représente le combat pour la laïcité, l'émancipation et la mixité et qui, par conséquent, se fait l'écho de ce qu'eux-mêmes cherchent à transmettre aux citoyens vivant sur le sol français. Un exemple qui l'atteste parce qu'il illustre parfaitement le rôle du féminin en politique et qui, de surcroît, n'est pas passé inaperçu dans l'opinion ${ }^{10}$ est celui du débat télévisé, 100 minutes pour convaincre, du 20 
novembre 2003 (O livier Mazerolle, Alain D uhamel, France 2), où N icolas Sarkozy, alors ministre de l'Intérieur, de la Sécurité intérieure et des Libertés locales, est invité à converser sur le thème de la laïcité, du port du voile et du rôle de l'islam dans la vie sociale française, avec Tariq Ramadan, islamologue et philosophe. En amont de l'échange, O livier Mazerolle ouvre la voie au ministre et lui demande son avis sur le " combat des ' $\mathrm{N}$ i putes ni soumises" ", lequel répond, sans détour, que " ces femmes ont parfaitement raison [et que] Madame Amara mène un combat remarquable ». Car, poursuit-il, " elles se plaignent de la disparition de l'état dans un certain nombre de quartiers qui sont devenus des ghettos, et où un certain nombre d'extrémistes et de délinquants font régner la terreur. [...]. L'État, hélas, par faiblesse, [a] déserté un certain nombre de cités et de quartiers ". Puis, il enchaîne sur une des thématiques développées dans les témoignages publiés dans Le Livre Blanc des Femmes de Quartier (Fédération nationale des Maisons des Potes, mars 2002), lorsqu'il rappelle que si « des filles mettent le voile, c'est pour qu'on les laisse tranquilles ". Sa réponse au processus de réclamation des " $\mathrm{Ni}$ putes ni soumises" correspond à ses fonctions du moment, puisqu'il souligne la présence indispensable de la police, destinée à " rétablir l'état de droit 》 dans ces « territoires de non-droit ". Sans conteste, cette réponse d'un ministre à une militante et ses sympathisants montre l'intérêt de certains membres du champ politique face à un mouvement et à " ce ou ceux " qu'il représente. D'autres exemples s'inscrivent dans cette perspective où il $\mathbf{y}$ a attirance du champ politique et médiatique masculin vers ce militantisme politique féminin (participation de représentantes de " $\mathrm{N}$ i putes ni soumises " à l'O bservatoire de la parité à la demande de $\mathrm{N}$ icole Ameline, ministre déléguée à la Parité et à l'Égalité professionnelle ; délégation officielle de plusieurs d'entre elles auprès du premier ministre, tean-Pierre Raffarin, et à la demande de ce dernier, etc.). Ceci est donc vérifiable et quantifiable. Et dès que faire se peut, politiques ou journalistes insèrent dans leur propre espace (meetings, débats télévisés, interviews dans la presse, etc.) certaines idées et propositions de " $\mathrm{Ni}$ putes ni soumises ", mais surtout citent ou glissent au détour de commentaires le nom de Fadela Amara et du mouvement, telle une référence indispensable et nécessaire à leur auto-représentation.

${ }^{10}$ Selon Médiamétrie, 6078480 téléspectateurs ont regardé l'émission, soit un taux d'audience de $23 \%$ avec un pic à 23 heures, soit 7730000 téléspectateurs ou $28 \%$. 
Fausse neutralité ou maladresse langagière ?

Par ailleurs, les paroles que Fadela Amara délivre en public servent à démontrer que le projet qu'elle porte se situe au-delà des hommes politiques et de leur appartenance à un courant de pensée, à un parti, à un syndicat, etc., qu'il s'ancre uniquement dans les valeurs communes de la République française. Ainsi avance-t-elle l'idée que cela autorise toute alliance, temporaire ou non, à partir du moment où elle ne met pas en péril les idéaux prônés : liber té, égalité, fraternité, mais encore mixité et laïcité. Pour exemple emblématique de ce point de vue, on peut citer la variété des étiquettes politiques des intervenants, invités à animer les universités des femmes de " $\mathrm{N}$ i putes ni soumises ", à D ourdan, les 3 , 4 et 5 octobre 2003 ou les 8,9 et 10 octobre 2004 :y figurent aussi bien des représentants de la majorité gouvernementale (e.g. Paul Benayoum, ministre délégué à la Ville et à la Rénovation urbaine ; N icole Ameline, ministre déléguée à la Parité et à l'Égalité professionnelle ; teannette Bougrab, porte-parole de I'UMP ; Corinne Lepage, ancien ministre de l'Environnement, gouvernement luppé, mai 1995) que de l'opposition (e.g. Malek Boutih, secrétaire national du Parti Socialiste chargé des questions de société ; Ulien Dray, porte-parole du Parti Socialiste).

A contrario, d'autres actions menées par " $\mathrm{Ni}$ putes ni soumises" peuvent desservir cette neutralité revendiquée. II en est ainsi pour le projet " L'été en chantier " (inciter les jeunes de la région île de France à équiper leur cité, sauvegarder le patrimoine, organiser la fête du quartier), mis en ligne sur les sites de la Fédération nationale des Maisons des Potes (http $/ /$ www.macite.net/home $/{ }^{11}$ ) et de " $\mathrm{Ni}$ putes ni soumises ", où la participation et le soutien de ulien Dray, viceprésident du conseil régional, sont développés et, de fait, posent question. $Q$ ue ce dernier soutienne une action en direction de la région où il officie est une démarche classique, mais que son nom et sa fonction apparaissent tels quels sur des sites associatifs militants en est une autre. Par ailleurs, lorsque Fadela Amara (2003:129) rend hommage à teanLouis Debré, en tant que président de l'Assemblée nationale, pour son " soutien actif et chaleureux » dans le cadre de l'exposition Mariannes d'aujourd'hui $^{2}$ sur le fronton du Palais Bourbon, certes, elle défend l'idée d'une République une et indivisible, mais elle ne contribue pas pour autant à la construction de la neutralité recherchée. Enfin, lorsqu'elle soutient la discrimination positive (Amara, 2003 : 138), là encore, elle s'engage en exprimant publiquement une position qui relève du débat

\footnotetext{
${ }^{11}$ Mis en ligne le 15 avril 2004.

${ }^{12}$ II s'agit d'une fresque composée de quatorze portraits de jeunes femmes des cités portant bonnet phrygien ou cocarde tricolore.
} 
politique et qui est loin d'être neutre : son opinion rejoint celle d'élus qui se sont exprimés sur le sujet, tels $\mathrm{N}$ icolas Sarkozy ou tean-Pierre Chevènement $^{13}$, pour ne citer qu'eux. $D$ 'autres exemples pourraient alimenter cette impossibilité frappante à vouloir à la fois défendre des idées transversales et communes à l'esprit républicain, présenter partiellement un projet de société tourné vers les femmes, puis les jeunes et enfin les hommes vivant dans les territoires des cités, et revendiquer - quoi qu'il advienne - une neutralité politique.

Depuis que Fadela Amara a franchi elle-même la ligne en invitant différents élus dans ses manifestations, en laissant citer son nom pour en appuyer d'autres, en prenant position sur des événements internationaux - les derniers en date étant l'appel du 30 août 2004 à une mobilisation générale pour la libération des deux journalistes français et de leur chauffeur syrien, otages en Irak, puis ses propos du 8 octobre $2004^{14}$ sur France-Info décrivant l'ampleur politique de " $\mathrm{Ni}$ putes ni soumises" et ses “ambitions d'européanité ", voire d'internationalisation - elle a montré ou fait la preuve qu'elle voulait intégrer l'espace politique. Dès lors, doit-elle en subir les exigences et les contraintes - entre autres celles des médias - qui, du fait qu'elle est devenue un personnage public, interviennent avec une résonance et un impact beaucoup plus forts ? II s'agit notamment de cette relation triangulaire d'interaction entre ce qu'elle dit à la presse en tant que porte-parole du mouvement, ce que les journalistes en disent, et ce que le champ politique - lui aussi - privilégie ou non (e.g. N icolas Sarkozy, ulien D ray). Et comme elle fait partie de ceux qui amènent " la société, qu'elle soit civile ou religieuse, à tenir compte de leur existence dans la définition des droits et devoirs des par tenaires sociaux (D agenais, 1999 : 14), elle est à la fois l'objet et l'acteur de véritables " relations presse ", par le biais, notamment, de ses attachés de presse travaillant pour les maisons d'éditions avec lesquelles elle a collaboré, comme La Découverte pour Ni putes ni soumises ${ }^{15}$ et les éditions Thierry Magnier pour sa préface d'un recueil de nouvelles intitulé Des filles et des garçons (2003). O n relèvera aussi son carnet personnel d'adresses qui compte celle de la journaliste Sylvia Z appi ou celles des journalistes du magazine Elle avec lesquelles elle a établi un partenariat d'actions ${ }^{16}$, depuis qu'elle

${ }^{13} \mathrm{~J}$-P. Chevènement avait institué un recrutement spécifique dans la police pour les jeunes des banlieues et issus de l'immigration.

${ }^{14}$ Ces propos ont été recueillis à l'occasion de l'ouverture de la deuxième université de "Ni putes ni soumises " à D ourdan, octobre 2004.

${ }^{15}$ Pour exemple, on peut citer les rencontres débats autour de son ouvrage (qui a obtenu le prix du Livre Politique 2004) annoncées par voie de presse dont celles avec J Minces, auteur de Le Coran et les femmes (2004).

${ }^{16}$ On notera, par exemple, le concours « Elle solidarité mode " d'avril 2004, réservé à des jeunes filles des cités et ayant pour fonction de leur faire intégrer " une [...] grande école de stylisme ». 
a signé leur pétition adressée au président de la République contre le port du voile islamique en décembre 2003. Enfin, on notera les noms de ceux et celles qui ont participé aux journées d'études de la dernière université de Dourdan, Laure Adler, directrice de France-Culture, teanFrançois Kahn, directeur de l'hebdomadaire Marianne, Valérie Toranian, directrice de la rédaction du magazine Elle, Rania El Baz, journaliste saoudienne et Aïcha $Z$ aïmi Sakhri, directrice de la rédaction du magazine Femmes du M aroc, et enfin tous les soutiens du monde du spectacle, des arts et des lettres. $\mathrm{Si}$ toutes ces stratégies publiques rendent visible l'association " $\mathrm{Ni}$ putes ni soumises ", elles ne contribuent pas pour autant à démontrer une neutralité clairement établie. Au vu de l'analyse, il semblerait qu'il y ait confusion entre l'idée de neutralité - qui n'existe pas dans ce cadre -, et la quête d'une forme d'indépendance politique.

\section{Conclusion}

O n peut se demander quels seront les effets, à moyen ou à long terme, des actions et revendications de cette association qui vise un autre espace que celui qui serait purement associatif, c'est-à-dire un espace politique avec tout ce que cela suppose : programme politique, présentation de candidats à de futures élections, etc. De fait, l'association “ Ni putes ni soumises" se comporte, d'ores et déjà, comme un mouvement politique qui prend corps. En tout cas, la question mérite d'être posée. En outre, nous ne nous trouvons plus dans ce que Fatiha Dazi-Heni et Catherine Polac (1990:62) appelaient «la situation dominée et marginale des femmes dans le mouvement associatif, [...] imputable [...] alors à la condition de filles d'immigrés mais aussi aux relations que la division sexuelle entretenait avec la distribution du travail militant ", même si, comme le rappellent Sylvie Pionchon et Grégory Derville (2004), il existe encore de fortes résistances des hommes politiques à l'égard des femmes politiques, quels que soient la structure d'engagement (collectif, association, parti politique) et le niveau (adhérent de base, militant de base, responsable associatif, politique, élu, etc.). Et c'est sans doute parce que « la présence féminine en politique n'est pas acquise au point d'apparaître banale et ordinaire [qu'elle] continue de faire question " (Pionchon, Derville, $2004: 96)$ que des femmes - telle Fadela Amara - en viennent à user de stratégies variées (celle de retournement de stigmate ; celle de l'enjeu avec le refus du port du voile ou de la lutte pour la discrimination positive ;celle du label “ Ni putes ni soumises »...) pour pouvoir pénétrer l'agora, se faire entendre et être porteuses de projets résolument politiques. 


\section{Références}

Abel O., 2004, « On se légitime par le malheur que l'on subit », Le Monde, 2223 août.

Amara F., Zappi S., 2003, Ni putes ni soumises, Paris, Éd. La D écouverte.

Badinter É., 1992, XY. De l'identité masculine, Paris, O. łcob, 2004.

- 2003, Fausse route, Paris, O. dacob.

Bard C., Baudelot C., Mossuz-Lavau J, 2004, Quand les femmes s'en mêlent. Genre et pouvoir, Paris, Éd. La Martinière.

Barthes Y., Callon M., Lascoumes P., 2001, Agir dans un monde incertain. Essai sur la démocratie technique, Paris, Éd. du Seuil.

Bellil S., 2002, Dans l'enfer des tournantes, Paris, D enoël.

Bonnafous S., 1999, "La médiatisation de la question immigrée : état des recherches ", Études de communication, 22, pp. 59-72.

Bouamama S., 1994, Dix ans de marche des beurs, Paris, EPI/D esclée de Brouwer.

Dagenais B., 1999, Le métier de relationnistes, $Q$ uébec, Presses de l'université Laval.

Dazi-Heni F., Polac C., 1990, « Chroniques de la 'vraie base". La constitution et les transformations du réseau associatif 'immigré" à $\mathrm{N}$ anterre ", Politix, 12, pp. 54-69.

Delorme C., 1985, Par amour et par colère, Paris, Éd. Le Centurion.

Des Filles et des garçons, 2003, recueil de nouvelles préfacées par F. Amara, Paris, T. Magnier.

Donzelot J, Mével C., 2002, “ La participation : entre construction et construction d'un pouvoir et accomplissement d'un devoir ", Lien social et politique, 48, pp. 81-93.

Favre, $P_{\text {. }}$ 1999, « Les manifestations de rue entre espace privé et espaces publics, pp. 135-152, in :François B., N eveu É., dirs, Espaces publics mosaïques. Acteurs, arènes et rhétoriques, des débats publics contemporains, Rennes, Presses universitaires de Rennes.

François B., Neveu É., 1999, «Pour une sociologie des espaces publics contemporains ", pp. 13-58, in : François B., Neveu É, dirs, Espaces publics mosaïques. Acteurs, arènes et rhétoriques, des débats publics contemporains, Rennes, Presses universitaires de Rennes.

Gastaut Y., 2000, L'immigration et l'opinion en France sous la Ve République, Paris, Éd. du Seuil.

Hily M.-A., Berthomière W., 2004, “ La notion de "réseaux sociaux" en migration », Hommes et migrations, 1250, juil.-août, pp. 6-12.

Malik S., 1990, H istoire secrète de SOS-Racisme, Paris, A. Michel.

Minces J, 2004, Le Coran et les femmes, Paris, Hachette Littératures. 
Mossuz-Lavau J,2004, “ Inégalités hommes/femmes :où en est-on ? ", Sciences humaines, hors-série, 46, pp. 20-24.

N eveu É., 1999, « L'approche constructiviste des 'problèmes publics". Un aperçu des travaux anglo-saxons ", Études de communication, 22, pp. 41-57.

Perrot M., 2001, Les femmes ou les silences de l'histoire, 5 vol., Paris, Flammarion.

Perrot M., Duby G., dirs, 1991, L'histoire des femmes en Occident, Paris, Perrin, 2002.

Pionchon S., Derville G., 2004, Les femmes et la politique, Grenoble, Presses universitaires de Grenoble.

Singly Fr. de, 2003, Les uns avec les autres. Quand l'individualisme crée du lien, Paris, A. Colin.

Thiéblemont-Dollet S., 2003, "Témoignages de femmes immigrantes et construction d'un problème public ", Questions de communication, 4, pp. 107-126. 